\title{
Non-fatal portal pyaemia complicating Crohn's disease of the terminal ileum
}

\author{
H Al-Jahdali, C Pon, W G Thompson, F R Matzinger
}

\begin{abstract}
A 40 year old woman with known Crohn's disease of the ileum but no abscess was found to have hepatic portal venous gas by computed tomography. Aggressive antibiotic treatment led to recovery and the ileum was resected two weeks later.

(Gut 1994; 35: 560-561)
\end{abstract}

Division of

Gastroenterology and Department of Radiological Sciences, Ottawa Civic Hospital, Ottawa, Canada H Al-Jahdali C Pon

W G Thompson

F R Matzinger

Correspondence to: DrW G Thompson, Division of Gastroenterology, Ottawa Civic Hospital, 1053 Carling Avenue, Ottawa, Ontario, Canada K1Y 4E9.

Accepted for publication 11 August 1993

Figure 1: Small bowel enema showing narrowed segment of terminal ileum with deep intramural fissures (arrows).
Air in the portal venous system points to portal pyaemia and usually predicts a fatal outcome. ${ }^{1}$ It results from a serious bowel disease, usually ischaemic necrosis. We report on a patient with acute ileal Crohn's disease who showed this phenomenon and survived.

\section{Case report}

A 40 year old woman had a 20 year history of Crohn's disease, which included an ileocaecal resection and in 1983 a revision of an ileal colic anastomosis. Despite known recurrence proxi-
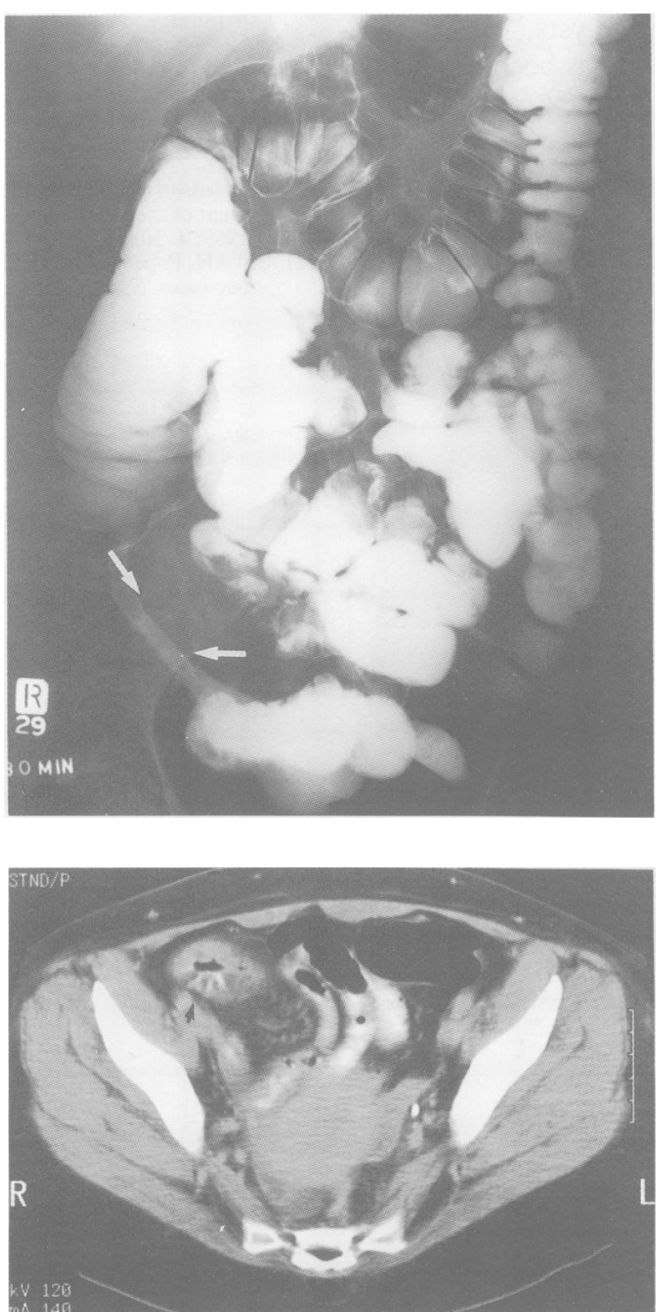

mal to the anastomosis, she suffered only minor symptoms related to iron deficiency. She was admitted 10 May 1992 with increasing weakness, malaise, abdominal cramps, and loose, watery, and sometimes black stools. Physical examination showed the surgical scar and a mass in the right lower quadrant. She was afebrile. The haemoglobin concentration was 103 and the white blood cell count was $11 \cdot 8$. The following day an ultrasound examination showed a segment of thickened terminal ileum, but no abscess or free fluid. Two days later a small bowel enema showed a considerably narrowed lumen of the neoterminal ileum with thickened walls and deep intramural fissures (Fig 1). There was no obstruction or fistula.

The patient was treated with intravenous methylprednisolone $20 \mathrm{mg}$ every 12 hours and oral metronidazole $50 \mathrm{mg}$ every eight hours. A liquid diet was begun and a normal diet was resumed by the date of discharge, 11 days later.

At home she continued on prednisone $30 \mathrm{mg} /$ day but was readmitted 3 June with abdominal pain, dizziness, chills, and nausea. She seemed ill and dehydrated. The physical examination was unchanged from the previous admission. The patient was afebrile and the pulse was 80 , blood pressure was $100 / 60$. The haemoglobin concentration was 111 , white blood cell count was $7 \cdot 4$, and albumin 28 . Methylprednisolone was restarted and food was withheld. Plain radiographs of the abdomen were normal.

Two days later she developed rigours with a temperature of $40.5^{\circ} \mathrm{C}$. Physical examination and a second abdominal ultrasound were unchanged. Chest $x$ ray was negative. Metronidazole $500 \mathrm{mg}$ every eight hours, ampicillin $500 \mathrm{mg}$ every eight hours, and gentamicin $80 \mathrm{mg}$ every eight hours were started intravenously but fever and rigours continued. The white blood cell count was $5 \cdot 4$, platelets 86 , and fibrin breakdown products were present.

Because of uncontrolled sepsis and evidence of early DIC, emergency computed tomography was performed. It showed a thickened terminal ileum with deep intramural fissures (Fig 2). Gas was seen in the periphery of the left lobe of the liver consistent with portal venous gas (Fig 3A). Diffuse intrahepatic periportal oedema was also present, and there was gas in the superior mesenteric vein. There was no free intraperitoneal fluid or abscesses.

Blood cultures grew Escherichia coli. The antibiotic was changed to imipenem $500 \mathrm{mg}$ every six hours and the fever resolved within two days. Repeat computed tomography on 11 June showed that the portal venous gas had disappeared (Fig 3B) and colour Doppler evaluation of the liver showed normal portal venous flow with no portal vein thrombosis. 

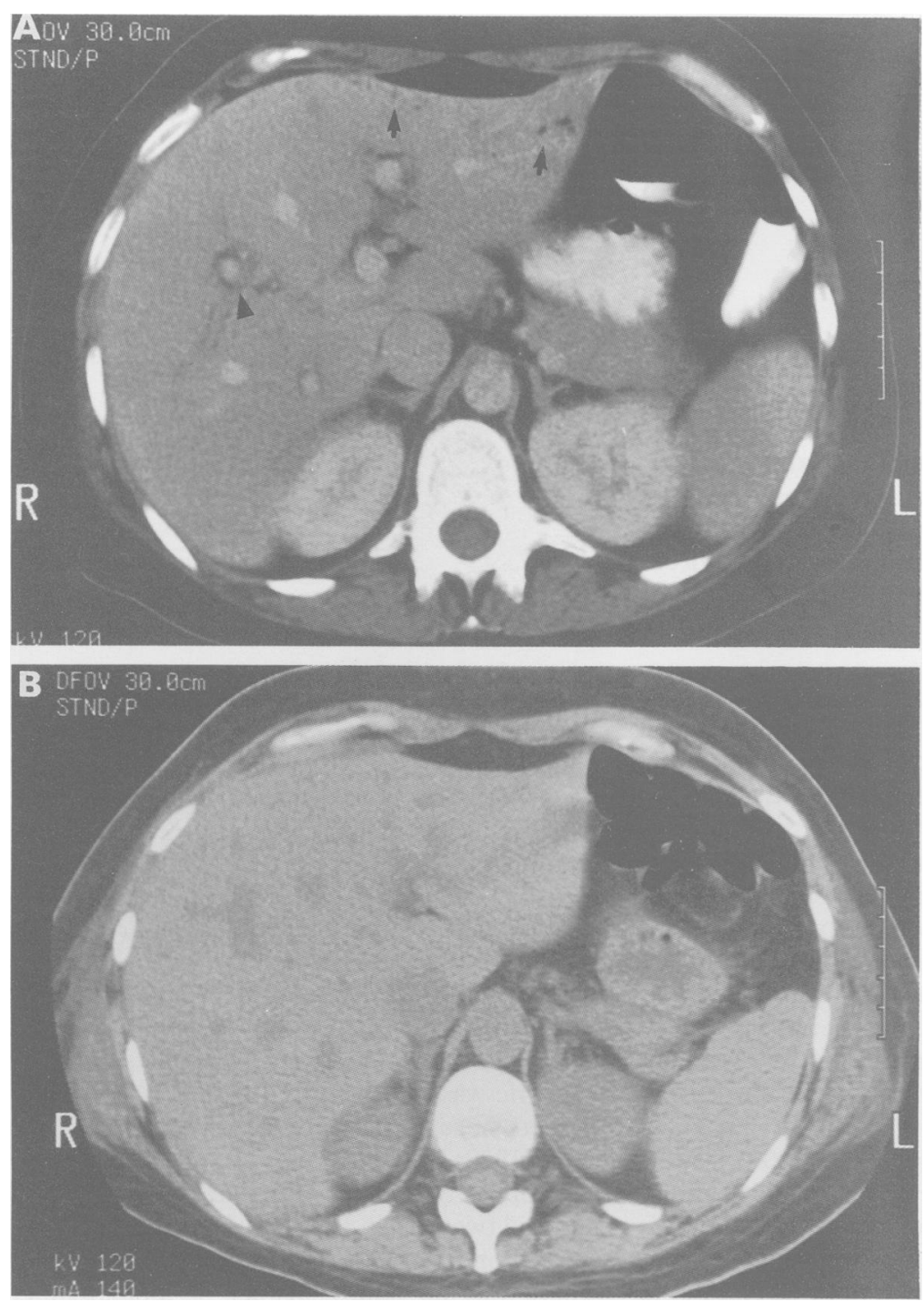

Figure 3: (A) Enhanced computed tomography shows gas in the periphery of the left lobe of the liver as a result of portal venous gas (arrow). The hypodense rims around the portal veins represent periportal oedema (arrowhead); (B) Follow up unenhanced computed tomography five days later, shows resolution of the portal venous gas.

Two weeks later a $15 \mathrm{~cm}$ resection of the neoterminal ileum was performed. The liver was normal at laparotomy. Pathological examination of the specimen confirmed Crohn's disease.

\section{Discussion}

Hepatoportal venous gas was first described in 1955 in infants with necrotising enterocolitis. ${ }^{2}$ There are two theories of its pathogenesis. One suggests that mesenteric and portal venous septicaemia results from a breach in the mucosa and invasion by gas forming organisms. This is the preferred theory in inflammatory processes and gangrene.' A second theory suggests that portal venous gas results from raised intraluminal pressure during considerable intestinal distension, which forces intraluminal gas into the intramural venous plexus through an ulcer, fissure or mucosal tear.'

Hepatoportal venous gas is considered to be a grave indication of bowel infarction or necrotising enterocolitis with a high death rate. ${ }^{12}$ It has also been described in blunt abdominal trauma, abscess, diverticulitis, granulomatous enterovenous fistula, and gastric ulcer. ${ }^{3-7}$

Recently, patients with considerable gastric or small bowel distension secondary to mechanical or functional obstruction have been found to have associated hepatoportal venous gas. ${ }^{8}$ These were successfully treated with antibiotics. Most cases of inflammatory bowel disease associated with hepatoportal venous gas that have been successfully managed with medical treatment were associated with a recent barium enema or colonoscopy. ${ }^{910}$ This invokes the second theory of pathogenesis.

Hepatoportal venous gas has been described twice before in Crohn's ileitis without abscess. In one fatal case, colonoscopy and double contrast barium enema had been performed 12 days before the detection of hepatoportal venous gas. "In the second case, hepatoportal venous gas was the initial radiological finding and the small bowel enema was performed after conservative treatment. ${ }^{12}$ These two cases and this case illustrate that inflammatory bowel disease without obstruction, abscess, or prior double contrast barium enema or endoscopy can lead to portal venous sepsis with hepatic portal venous gas. Initial aggressive antibiotic treatment followed by surgical resection can prevent a fatal outcome.

1 Liebman PR, Patten MT, Manny J, et al. Hepatic portal venous gas in adults: etiology, pathophysiology and clinical significance. Ann Surg 1978; 187: 281 .

2 Wolfe JN, Evans WA. Gas in the portal veins of the liver in infants: a roentgenographic demonstration with post infants: a roentgenographic demonstration with

3 Friedman D, Flancbaum L, Ritter E, Trooskin SZ. Hepatic portal venous gas identified by computed tomography in a patient with blunt abdominal trauma: a case report. F Trauma 1991; 31: 290-2.

4 Ajzen SA, Gibney RG, Cooperberg PL, et al. Enterovenous fistula: unusual complication of Crohn's disease. Radiology 1988; 166: 745-6.

5 Bach MC, Anderson LG, Martin TA, et al. Gas in the hepatic portal venous system: a diagnostic clue to an occult intraabdominal abscess. Arch Intern Med 1982; 142: 1725-6.

6 Graham GA, Berstein RB, Granner AT. Gas in the portal and inferior mesenteric veins caused by diverticulitis of the sigmoid colon. Radiology 1975; 114: 601-2.

7 Edwards AM, Michalyshyn B, Costopoulos LB. Survival following gas in the portal venous system: a report of two following gas in the portal venous system:

9 Celoria G, Coe N. Does the presence of hepatic portal venous gas mandate an operation? A reassessment. South Med $\mathcal{F}$ 1990; 83: $592-4$

9 Kees CJ, Hester CC. Portal vein gas following barium enema examination. Radiology 1972; 102: 525.

10 Haber I. Hepatic portal vein gas following colonoscopy in ulcerative colitis: report of a case. Acta Gastroenterol Belg 1983; 46: 14.

11 Delamarre J, Capron JP, et al. Spontaneous portal venous gas in a patient with Crohn's ileocolitis. Gastrointest Radiol 1991; 16: $38-40$.

12 Kirsh M, Bozdech J, Gardner DA. Hepatic portal venous gas: an unusual presentation of Crohn's disease. Am f Gastroenterol 1990 ; 85: 1521-3. 